\title{
Transscleral Removal of Subretinal Strand without Vitrectomy: A Case Report
}

\author{
Yongping Tang ${ }^{\mathrm{a}, \mathrm{b}}$ Ronghan $\mathrm{Wu}^{\mathrm{a}, \mathrm{b}}$ \\ aEye Hospital and School of Ophthalmology and Optometry, Wenzhou Medical University, \\ Wenzhou, China; ${ }^{b}$ National Clinical Research Centre for Ocular Diseases, Wenzhou, China
}

\section{Keywords}

Retinal detachment · Scleral buckling · Transscleral removal · Silicone oil tamponade ·

Subretinal strand

\begin{abstract}
Recurrence of inferior retinal detachment, after vitreoretinal surgery and silicone oil tamponade, along with a subretinal strand, presents a challenge for surgeons. Vitrectomy and retinotomy are the usual treatment in such cases. Here, we present a new transscleral method for addressing this problem. A 13-year-old boy with recurrent retinal detachment after silicone oil tamponade underwent scleral buckling surgery and had a transscleral subretinal strand removed without retinotomy. The retina reattached, and silicone oil was removed 3 months later. The best-corrected visual acuity was 0.4 in decimal vision at 1 year after silicone oil removal. Scleral buckling surgery combined with subretinal strand removal may be used as an alternative to retinotomy, especially for patients with an inferior retinal detachment and local subretinal strand formation.
\end{abstract}

\section{Introduction}

A variety of methods are used to treat retinal detachment (RD), such as pneumatic retinopexy, scleral buckling (SB), and pars plana vitrectomy (PPV) combined with intraocular tamponade [1]. Although the surgical techniques and equipment have significantly improved over time, RD with an inferior break is still a challenging surgical problem. Because of the low density and high buoyancy force, conventional silicone oils (SO) (and gases) are relatively less 
effective in the treatment of inferior RD [2]. Furthermore, SO tamponade left in the eye during a PPV for inferior RD may lead to the sequestration of retinal pigment epithelium cells over the inferior retina, resulting in proliferative vitreoretinopathy (PVR), which remains the principal cause of failed surgery for RD [3, 4]. Recurrent RD with retinal traction, retinal shortening, or subretinal proliferative strand formation is usually treated by vitrectomy and retinotomy, which may necessitate multiple surgeries to reattach the retina [5]. Retinotomy is a useful surgical technique in the management of complex RD with PVR when other procedures, including membrane peeling and SB, have failed. However, creating the retinotomy, as well as removing membranes through this retinotomy, can increase the incidence of unhealed retinal break, PVR on the posterior free edge, and postoperative hypotony. Thus, the use of retinotomy requires careful evaluation [6]. Wolff [7] reported a new method to remove a subretinal strand via a scleral canal during SB in a young patient and achieved complete retinal reattachment at day 1 . It was indeed a novel, less-invasive treatment for a young patient with RD and PVR.

Here, we describe the case of a 13-year-boy who had PPV surgery combined with SO tamponade for an RD in the right eye; 3 months later, an inferior RD with subretinal strand was found. The patient and his parents refused another vitrectomy and could not ensure strict prone positioning. We successfully removed the subretinal strand via a transscleral canal and reattached the retina by SB.

\section{Case Presentation}

A 13-year-old boy was referred to our clinic with the recurrence of RD in his right eye, after having undergone a PPV and SO tamponade because of RD in that eye 3 months earlier in another hospital. No details of the first surgery were provided. At the first visit to our clinic, the patient's visual acuity was 0.3 in decimal vision, with myopia of 3 diopters in the right eye. Axial length was $27.51 \mathrm{~mm}$. Intraocular pressure was $3.2 \mathrm{~mm} \mathrm{Hg}$. Slit-lamp examination revealed that the cornea and lens were both transparent, and the vitreous cavity was filled with SO. The fundus was examined carefully: the disc border was clear with a $1 / 2$-papillary diameter (PD) arc of atrophy on the temporal side, and the cup-to-disc ratio was within normal limits. Inferior RD was found with a 1/2-PD round hole at 8:30 o'clock of the detached retina. The detached retina was stiff, with a subretinal strand formed. The fundus of the patient's left eye was also checked. The left eye disc border was clear with a $1 / 2$-PD atrophy arc on the temporal side, the cup-to-disc ratio was approximately 0.3 , a degeneration zone was seen in the peripheral retina, in the temporal quadrant, and the retina was attached without holes or breaks. Optical coherence tomography (OCT) scans (Heidelberg Engineering, Heidelberg, Germany) showed that the macula in each eye remained attached. The patient denied any history of trauma or relevant family history. According to his fundus examination, symmetrical peripheral retinal degeneration without any premature or oxygen-inhaling history, we thought this patient might have familial exudative vitreoretinopathy, while the patient and his parents refused to do fluorescein fundus angiography or genetic test.

The details in this case made it problematic: a young patient presented with an unsealed inferior retina break, stiff retina, and significant subretinal strand and had already undergone a PPV with SO tamponade. For this patient, retinotomy after SO removal would create a new break and need SO tamponade again. The rate of recurrent RD will be high because the break and retinotomy were both in the inferior quadrant. Another surgery to remove SO will be needed too. The second choice was SB combined with retinotomy and SO tamponade which might achieve anatomic success, without improving the visual outcome, especially in a young

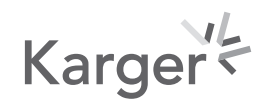




\section{Case Reports in Ophthalmology}
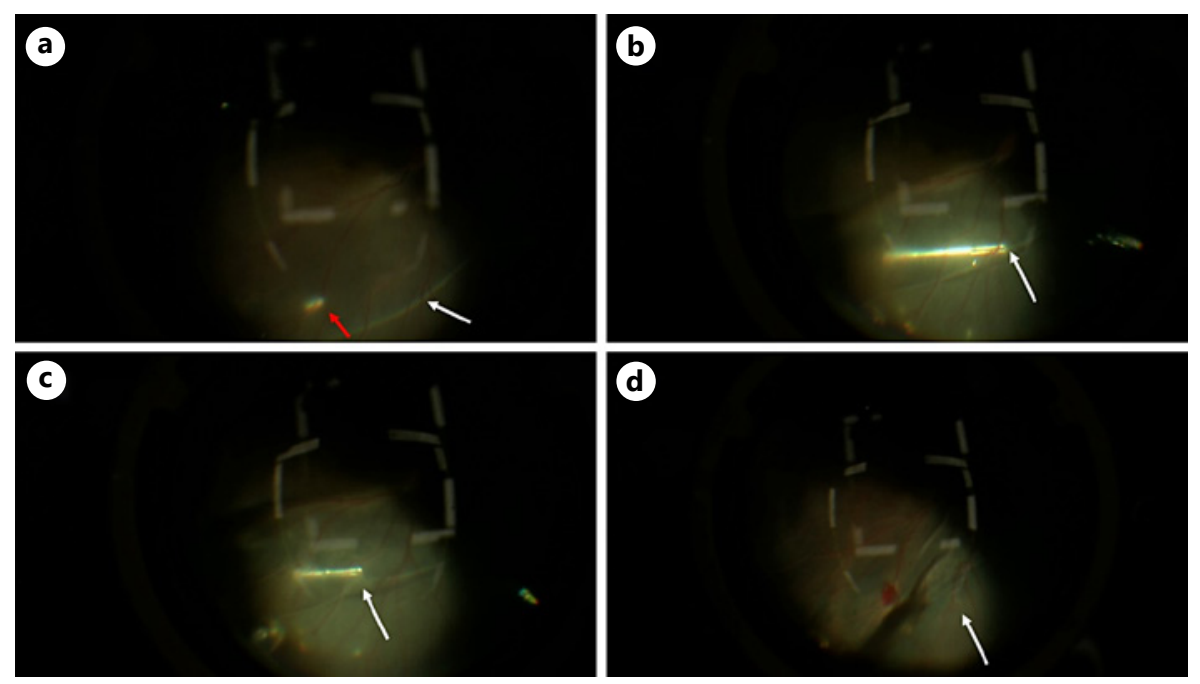

Fig. 1. a The trocar was inserted into the subretinal space (red arrow), and the location of the subretinal proliferative strand (white arrow) was determined. b, c The subretinal strand was grasped and removed with vitreoretinal forceps (white arrow). d The fundus after the silicon sponge was placed.

patient. Solaiman and Dabour [3] found that for selected cases of inferior RDs in SO-filled eyes, supplemental SB could be as effective as a second vitreoretinal surgery. However, SB alone might not have been sufficient to reattach the retina of this patient because of the subretinal strand. Wolff [7] removed a subretinal strand via a scleral canal during SB in a young patient and achieved complete retinal reattachment. Therefore, we chose SB surgery with transscleral removal of the subretinal strand for this patient.

After the patient was under general anesthesia, cryopexy was performed, and a segmental buckle was placed in the standard manner. Retinal visualization was established with a 25-gauge illuminator (Alcon Laboratories, Fort Worth, TX, USA) and noncontact wide-angle viewing system (Resight ${ }^{\mathrm{TM}}$; Carl Zeiss Meditec AG, Jena, Germany). Examination of the fundus of the right eye showed RD from 3 to 9 o'clock in the periphery, with inferior retina stiffness and a yellow-white, strip-like subretinal strand that ranged from 5 to 8 o' clock in the peripheral retina. A break of about $1 / 2$-PD was found at 8 o'clock of the equatorial retina. After locating the detached area, subretinal strand, and break, another transscleral canal was made with a 25-gauge trocar at 6-o'clock equator of the eyeball, through which a small amount of viscoelastic agent was injected into the subretinal space, to enlarge the operating space (Fig. 1a). Vitreoretinal forceps were inserted into the subretinal space through the inferior cannula, to grasp and remove the subretinal strand (Fig. 1b, c). Then, the cannula was removed. Cryotherapy was applied after drainage of SRF. A silicon sponge was placed on the scleral surface over the corresponding region. The fundus was checked, and the break was located on the crest of the sponge, with the stiff retina reattached to the choroid membranes (Fig. 1d). About $0.5 \mathrm{~mL}$ of sterile air was injected into the vitreous cavity. The intraocular pressure was normal at the end of surgery (the brief surgical process is shown in the video).

Slit-lamp examination, visual acuity, intraocular pressure, fundus examination, and OCT scans were performed at 1 week and every month postoperatively. No subretinal proliferative strand was found in the fundus examination after surgery (Fig. 2). OCT scans indicated that the subretinal fluid was absorbed and the retina reattached gradually (Fig. 3). Three months later, the SO in the right eye was removed. The retina remained attached, and the best-corrected visual acuity was 0.4 in decimal vision, 1 year after SO removal. However, the proliferative membrane was found in the peripheral degenerative

\section{Karger'}




\section{Case Reports in Ophthalmology}
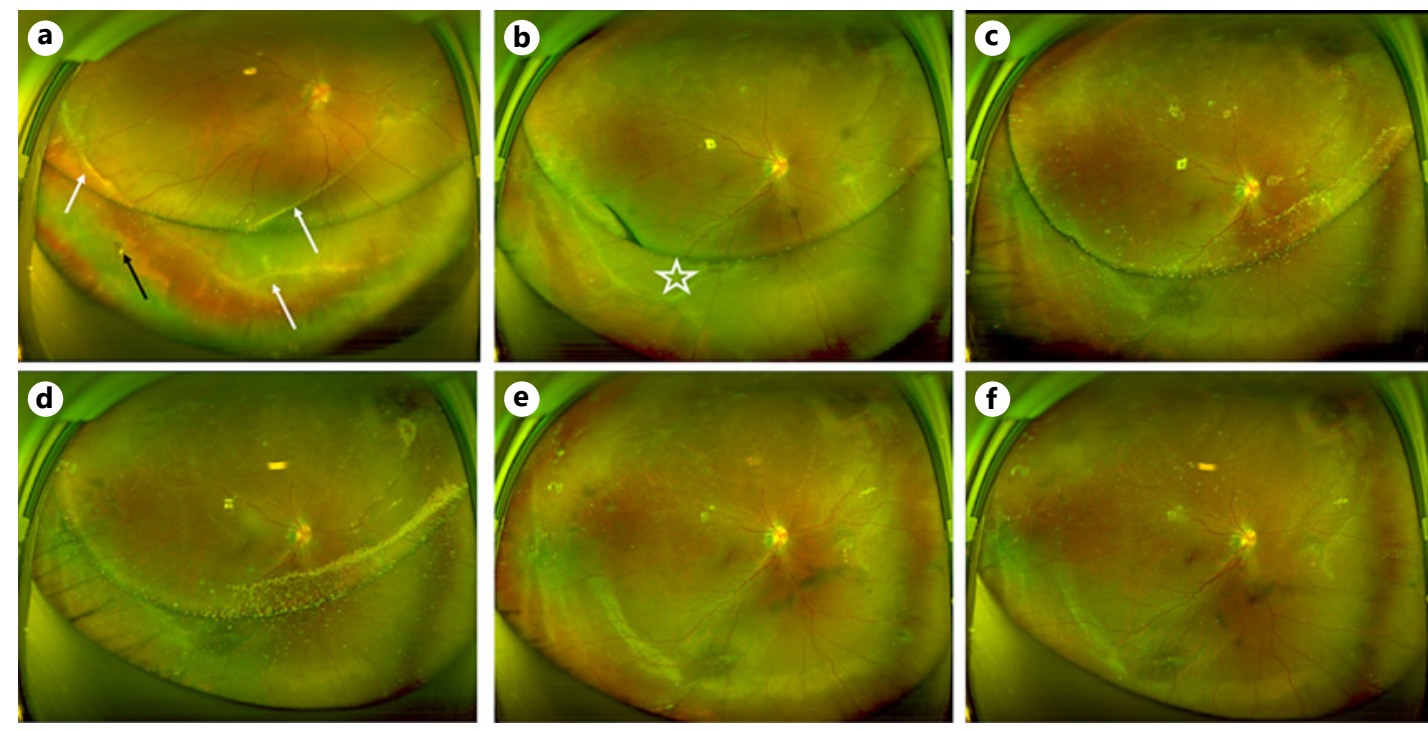

Fig. 2. a The fundus of right eye before the scleral buckle surgery, in which subretinal strand (white arrows) and a retinal hole (black arrow) could be seen clearly. $\mathbf{b}$ In the fundus of the right eye, examined 1 week postoperatively, no subretinal strands were seen, the buckle was set in the proper position, and the buckle height was adequate (white star); a small amount of subretinal fluid was found at the peripheral edge of the buckle. c In this fundus image of the right eye, 1 month postoperatively, emulsification of the silicone oil was seen, and the retina remained attached. $\mathbf{d}$ In this fundus image of the right eye, examined 3 months postoperatively, more emulsification of the silicone oil was seen than in the previous examination. e, $\mathbf{f}$ Fundus images of the right eye, examined 1 month and 1 year after the silicone oil removal.

zone of his left eye during the recent follow-up, which showed a clinic feature of familial exudative vitreoretinopathy.

\section{Discussion and Conclusions}

In this case, the patient was young, and an unsealed inferior break and subretinal strand were found after the primary PPV with SO tamponade. These factors together made this recurrent RD a challenging case. Luckily, the detached inferior retina had shrunk slightly, without obvious shortening, and it could be categorized to PVR stage C1. The surgical approach for such cases is repeated PPV with SO removal, elimination of traction via relaxing retinotomy and retinectomy, removal of the subretinal strand, use of perfluorocarbon liquids, reattachment of the retina, and SO or gas tamponade at the end of surgery [8, 9]. Alternatively, some surgeons have reported the use of SB combined with vitreoretinal surgery for treatment of such cases [10]. SB can reduce the distance between the inferior retina and the SO bubble, by reducing the volume of the vitreous cavity, and increase the tamponade effect of the SO. At the same time, the subretinal fluid is properly drained during surgery, to promote retinal reattachment and support the retinal breaks. Also, the SB theoretically floats the proliferative cells and the mediators of cellular proliferation away from the detached inferior retina in the upright position and thus decreases PVR and, consequently, decreases the rate of repeated inferior RD [11]. Solaiman and Dabour [3] reported that inferior RD in SO-filled eyes treated with supplemental SB and external drainage of subretinal fluid had satisfactory functional and anatomic outcomes in selected cases and could offer a faster, less-invasive, and less-expensive alternative to 


\section{Case Reports in Ophthalmology}

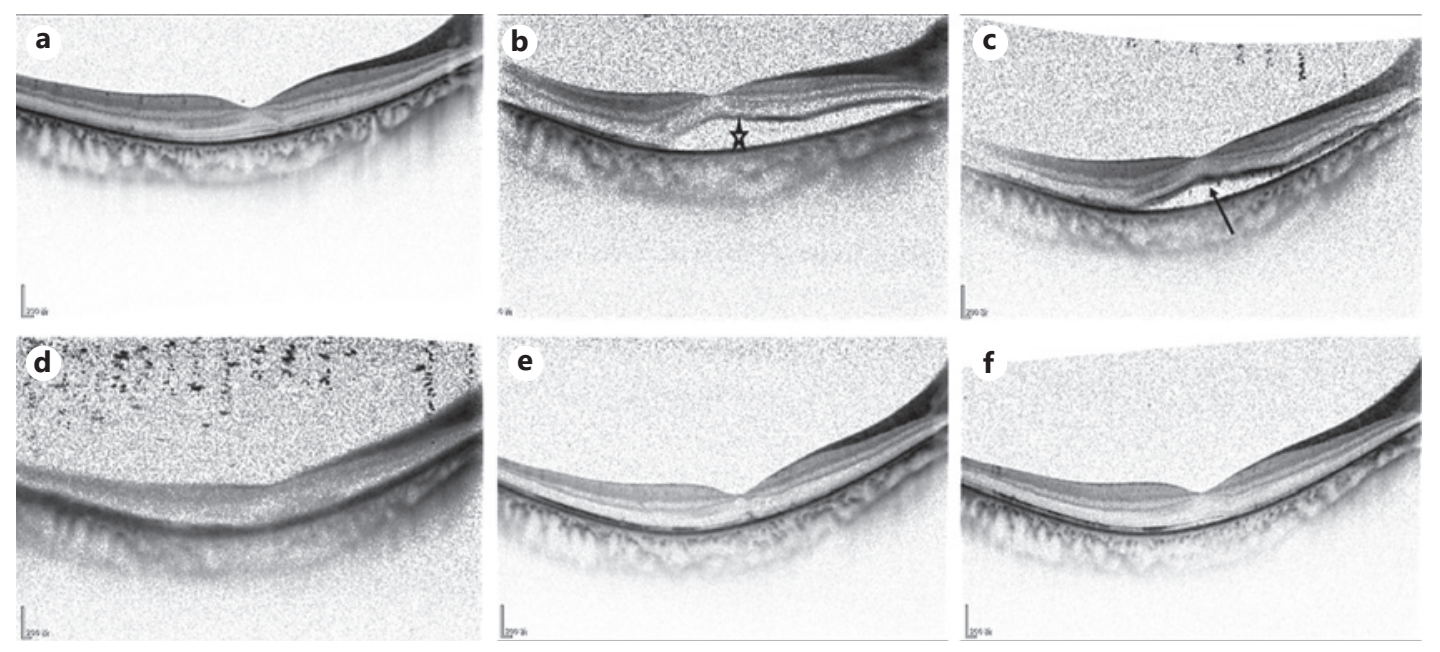

Fig. 3. a OCT scan of the patient's right eye before the SB surgery. b OCT results of the right eye examined 1 week postoperatively, with subretinal fluid visible at the macula (black star). c OCT from 1 month after the SB surgery, with subretinal fluid reduced relative to that of 1 week (black arrow). d In the OCT of the right eye 3 months postoperatively, no subretinal fluid was seen, and the retina was not clearly distinguishable with emulsification of the silicone oil. e, $\mathbf{f}$ OCT images of the right eye, 1 month and 1 year after the silicone oil removal surgery. OCT, optical coherence tomography; SB, scleral buckling.

repeated PPV for such re-RD cases with SO tamponade. Wei et al. [12] compared the effectiveness of SB versus re-vitrectomy according the time of re-detachment after primary surgery. Their study suggested that, for eyes with recurrent inferior RD under SO tamponade in the early period ( $\leq 1$ month) after primary PPV, SB might be an alternative because it caused fewer complications. Wolff [7] reported a novel, less-invasive way to remove the subretinal strand via scleral canal during SB for young RD patients with PVR instead of retinectomy and SO tamponade.

Therefore, we chose SB surgery and transscleral removal of the subretinal strand to seal the break and reattach the retina for this young patient. In his case, the retina reattached, and SO was removed 3 months later. During the regular follow-up, the retina stayed attached, and the best-corrected visual acuity was 0.4 in decimal vision at the last follow-up. Except for a short-term macular detachment after SB surgery, no sight-threatening complications, such as subretinal hemorrhage, retinal injury, intraocular hypertension, or recurrent RD, occurred in the operated eye. This procedure provides a faster and less-invasive way to deal with a subretinal strand and achieve good anatomic success. The transscleral removal of a subretinal strand reduces the number of operations needed and avoids the visual impairment caused by multiple operations, at the same time reducing the economic burden on patients. However, the state of the retina should be carefully evaluated before choosing this procedure. Patients with severe PVR, significant epiretinal membrane, prominent retinal shortening, and resolved retinal folds should be excluded.

\section{Acknowledgments}

The authors wish to thank Xiaoxiao Wang and Beilei Wu for collecting and organizing the image data and Tianyu Chen for reviewing the manuscript. They thank Claire Barnes, PhD, from Liwen Bianji, Edanz Editing China (www.liwenbianji.cn/ac), for editing the English text of a draft of this manuscript. 


\section{Statement of Ethics}

This report does not contain any personal information that could lead to the identification of the patient. However, written consent was obtained from patient's parents in the study, and the parents had given their written informed consent to publish this case. This study was approved by the Ethics Committee of the Eye Hospital of Wenzhou Medical University (2019-189-K-170).

\section{Conflict of Interest Statement}

The authors have no conflicts of interest to declare.

\section{Funding Sources}

This manuscript did not receive any funding.

\section{Author Contributions}

Ronghan Wu designed the study; Yongping Tang conducted the study and prepared the manuscript; both authors have read and approved the manuscript.

\section{Data Availability Statement}

All data analyzed during this study are included in this article and its supplementary material files. Further enquiries can be directed to the corresponding author.

\section{References}

1 Schwartz SG, Flynn HW Jr, Mieler WF. Update on retinal detachment surgery. Curr Opin Ophthalmol. 2013; 24(3):255-61.

2 Wetterqvist C, Wong D, Williams R, Stappler T, Herbert E, Freeburn S. Tamponade efficiency of perfluorohexyloctane and silicone oil solutions in a model eye chamber. Br J Ophthalmol. 2004;88:692-6.

3 Solaiman KA, Dabour SA. Supplemental scleral buckling for inferior retinal detachment in silicone oil-filled eyes. Retina. 2014 Jun;34(6):1076-82.

4 Charles S, Randolph JC, Sigler EJ. Pars plana vitrectomy reoperation without removing silicone oil. Retina. 2012;32(8):1664-5.

5 Quiram PA, Gonzales CR, Hu W, Gupta A, Yoshizumi MO, Kreiger AE, et al. Outcomes of vitrectomy with inferior retinectomy in patients with recurrent rhegmatogenous retinal detachments and proliferative vitreoretinopathy. Ophthalmology. 2006;113:2041-7.

6 Hocaoglu M, Karacorlu M, Muslubas IS, Ozdemir H, Arf S, Uysal O. Peripheral 360 degree retinotomy, anterior flap retinectomy, and radial retinotomy in the management of complex retinal detachment. Am J Ophthalmol. 2016 Mar;163:115-21.e1.

7 Wolff B. Subretinal surgery Ab Externo: a novel approach to access the subretinal space without the need for retinotomy. Retina. 2015 Jul;35(7):1474-5.

8 Mancino R, Aiello F, Ciuffoletti E, Di Carlo E, Cerulli A, Nucci C. Inferior retinotomy and silicone oil tamponade for recurrent inferior retinal detachment and grade C PVR in eyes previously treated with pars plana vitrectomy or scleral buckle. BMC Ophthalmol. 2015 Dec 9;15:173.

9 Sharma T, Gopal L, Shanmugam MP, Bhende PS, Agrawal R, Badrinath SS, et al. Management of recurrent retinal detachment in silicone oil-filled eyes. Retina. 2002;22:153-7.

10 Mester U, Anterist N, Kroll P, Brieden-Azvedo S. The role of the vitreous in retinal detachment surgery with external buckling. Ophthalmologica. 2002;216:242-5. 


\section{Case Reports in Ophthalmology}

11 Rush R, Sheth S, Surka S, Ho I, Gregory-Roberts J. Postoperative perfluoro-N-octane tamponade for primary retinal detachment repair. Retina. 2012;32:1114-20.

12 Wei Y, Wu G, Xu K, Wang J, Zu Z, Wang R. The outcomes of scleral buckling versus re-vitrectomy for the treatment of recurrent inferior retinal detachment in silicone oil tamponade eyes. Acta Ophthalmol. 2016 Nov; 94(7):e624-8. 